\title{
Efeito da Utilização de Bandas Elásticas \\ Durante Aulas de Hidroginástica na Força \\ Muscular de Mulheres
}

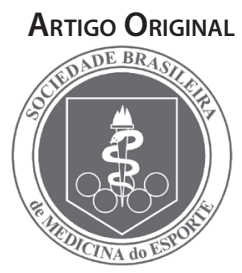

\author{
Effect of the Use of Elastic Bands During Water Gymnastics Classes in \\ Muscular Strength of Women
}

Luiz Gustavo Pinto'

Raphael Mendes Ritti Dias'2, 3,4

Emanuel Péricles Salvador 2, 3,4

Aylton Figueira Júnior ${ }^{1}$

Celimara Valente Gambá Lima`

1. Universidade Metodista de São Paulo - Faculdade de Educação

Física e Fisioterapia. São Bernardo do Campo, SP.

2. Grupo de Estudo e Pesquisa em Metabolismo, Nutrição e Exercício - Centro de Educação Física e

Desporto - Universidade Estadual de Londrina. Londrina, PR.

3. Programa de Pós-graduação em Saúde Pública - Universidade de São Paulo - Faculdade de Saúde

Pública. São Paulo, SP.

4. Bolsa Fapesp.

Endereço para correspondência: Luiz Gustavo Pinto

Rua Heizo Nakano, 44, ap. 31, bl 7

- Bairro Jerusalém - CEP 09811-370

São Bernardo do Campo, SP.

E-mail: luizgustavopinto@bol.com.br

Submetido em 14/08/2006

Versão final recebida em 24/11/2006

Aceito em 09/12/2006

\begin{abstract}
RESUMO
O objetivo deste estudo foi comparar as modificações na força/resistência muscular após quatro semanas de hidroginástica com e sem a utilização de bandas elásticas. Participaram do estudo 26 mulheres com idade entre 50 e 60 anos, praticantes de hidroginástica havia pelo menos seis meses. Pré e pós-treinamento, os sujeitos foram submetidos aos testes de flexão de cotovelo, impulsão vertical sem auxílio dos braços e levantar da cadeira em 30 segundos. Os sujeitos foram divididos aleatoriamente em dois grupos: treinamento elástico (GTE) e treinamento convencional (GTC). O GTE realizou a aula de hidroginástica com incremento de banda elástica como sobrecarga adicional. O GTC realizou aula de hidroginástica sem sobrecarga adicional. Para o tratamento estatístico foi utilizada análise de variância (ANOVA) por dois fatores, seguida do teste post hoc de Tukey com $\mathrm{P}<0,05$. Os resultados indicaram que houve efeito significante do tempo nos três testes analisados $(P<0,01)$. Adicionalmente, nos testes de flexão de cotovelo e levantar e sentar da cadeira foi verificado efeito significante da interação grupo x tempo $(P<0,01)$. O teste post hoc de Tukey indicou que, nos três testes, houve aumento significante do pré ao pós-treinamento apenas no GTE (flexão de cotovelo: +45,9\%; impulsão vertical: +31,7\%; e levantar e sentar da cadeira: 32,4\%). Esses resultados sugerem que a associação do treinamento de força realizado por meio de elásticos à hidroginástica promove aumentos significantes na força muscular de membros inferiores e superiores de mulheres. Dessa forma, por se tratar de um método versátil e viável economicamente, recomenda-se a incorporação de exercícios com elástico durante as sessões de hidroginástica para o aumento da força muscular em mulheres.
\end{abstract}

Palavras-chave: resistência elástica, atividades aquáticas, envelhecimento, aptidão física.

\section{ABSTRACT}

The purpose of this study was to compare the muscle strength/resistance changes after four weeks of water gymnastics with and without the use of elastic bands. 26 women aged 50-60 years, who have attended water gymnastics classes for at least six month participated in the study. The subjects were pre and post-training assessed through elbow flexion, vertical jump without arm movements and 30-second stand and seat chair tests. The sample was randomly divided into two groups: Elastic band group (EBG) and Regular training group (RTG). EBG was engaged in water gymnastics classes program with the elastic band as additional load, while GTC engaged in only regular water gymnastics classes with no additional apparatus. The statistic analysis chosen was the two-way ANOVA and Tukey post hoc test with $p<0.05$ as significant level. The results presented positive effect of the training program in the three analyzed variables. Elbow flexion and chair stand and seat tests showed significant in the group $x$ time interaction $(p>0.01$ ). Tukey post hoc test evidenced significant increase pre-post training period at EBG program for all studied variables. These results suggest that elastic bands are an interesting strategy for water gymnastics classes to increase muscle strength of upper and lower limbs in women.

Keywords: elastic bands resistance, water activities, aging, physical fitness. 


\section{INTRODUÇÃO}

Embora o crescimento da população idosa mundial seja um importante indicativo da melhoria da qualidade de vida dessa população, é bem conhecido que o processo de envelhecimento está atrelado a perdas importantes em inúmeras capacidades físicas, as quais culminam, inevitavelmente, no declínio da capacidade funcional e da independência do idoso ${ }^{(1)}$. Exemplo disso são os achados de Frontera et al. (2), que observaram em idosos, por meio de estudo longitudinal, declínio anual de força entre 2,0 a 2,5\%, para membros inferiores.

Dessa forma, com o objetivo de minimizar ou reverter a diminuição da força com o envelhecimento, o posicionamento do American College of Sports Medicine ${ }^{(3)}$ sobre os benefícios do exercício físico para idosos recomenda a inclusão do treinamento de força nos programas de exercícios dessa população.

Nesse sentido, um dos métodos de treinamento de força que se tem tornado popular devido aos resultados positivos encontrados na literatura é o treinamento com bandas elásticas. Esse método apresenta como principais vantagens a versatilidade, a viabilidade econômica ${ }^{(4)}$ e a possibilidade de incrementar a produção de força durante o movimento ${ }^{(5)}$. o que têm feito com que seja utilizado em diferentes populações, que incluem tanto atletas, para a melhoria do desempenho esportivo( ${ }^{(5)}$, como idosos $^{(6,7)}$ e cardiopatas ${ }^{(8)}$, com o objetivo de melhoria da aptidão física relacionada à saúde.

Todavia, entre adultos e idosos, uma das modalidades mais praticadas para a melhoria da aptidão física é a hidroginástica. Diferentes estudos têm demonstrando benefícios fisiológicos promovidos pela hidroginástica, como aumento do $\mathrm{VO}_{2 \text { pico }}{ }^{(9)}$, da flexibilidade ${ }^{(10)}$ e da força muscular, ressaltando o estímulo do exercício na água ${ }^{(11)}$. Seria importante apresentar que, em função de o peso corporal não ser uma fator limitante para a prática da atividade na água, a aderência em programas de exercícios tende a aumentar. Contudo, os estudos são controversos em relação à força muscular. Enquanto dois estudos verificaram aumentos significantes após o treinamento de hidroginástica ${ }^{(9,10)}$, um não encontrou alterações significantes nessa capacidade ${ }^{(11)}$.

Sendo assim, a utilização de métodos que permitam potencializar os ganhos de força durante a hidroginástica consiste numa estratégia importante para a melhoria da aptidão física dos praticantes dessa modalidade. Assim, o objetivo do presente estudo foi comparar as modificações na força e resistência muscular após quatro semanas de hidroginástica com e sem a utilização de elásticos para o aumento da sobrecarga muscular.

\section{METODOLOGIA}

\section{Amostra}

Fizeram parte da amostra 26 mulheres com idade entre 50 e 60 anos (55,0 $\pm 2,4$ anos), alunas de uma academia na cidade de São Bernardo do Campo. As alunas faziam parte de duas turmas de hidroginástica da academia e realizavam duas sessões semanais de 50 minutos havia pelo menos seis meses, no mesmo horário, porém em dias diferentes (segunda e quarta ou terça e quinta-feiras).

Como critérios de inclusão ao estudo, os sujeitos não deveriam realizar outra modalidade de exercício físico e ter idade entre 50 e 60 anos. Os sujeitos que apresentaram mais de 25\% de ausência nas sessões de treinamento no decorrer do experimento foram excluídos das análises.

Previamente ao início da pesquisa, os sujeitos foram devidamente esclarecidos sobre os objetivos e procedimentos aos quais seriam submetidos, assinando o Termo de Consentimento Livre e Esclarecido no momento da apresentação metodológica do estudo. Este estudo foi aprovado pelo o Comitê de Ética em Pesquisa com Seres Humanos da instituição dos autores.

\section{Programa de treinamento e modelo experimental}

Previamente ao início do programa de treinamento, a amostra foi divida em dois grupos: treinamento elástico (GTE) e treinamento convencional (GTC). A divisão foi feita de modo aleatório, considerando os dias nos quais as aulas de hidroginástica eram realizadas. Assim, a turma que realizava aulas na segunda e quarta-feiras recebeu um tipo de intervenção, ao passo que a que fazia aulas nas terças e sextas-feiras recebeu outro tipo de intervenção. Para determinar a distribuição do tipo de intervenção (GTE ou GTC) por turma, foi realizado um sorteio. O GTE e o GTC foram compostos por 15 sujeitos em cada grupo. As características gerais dos grupos GTE e GTC no momento pré-treinamento estão apresentados na tabela 1.

Tabela 1. Média e desvio-padrão da idade, peso e estatura do GTE e GTC no momento pré-treinamento.

\begin{tabular}{lcc}
\hline & $\begin{array}{c}\text { GTE } \\
(\mathbf{n}=\mathbf{1 5})\end{array}$ & $\begin{array}{c}\text { GTC } \\
(\mathbf{n}=\mathbf{1 1 )}\end{array}$ \\
\hline Idade (anos) & $54,9 \pm 2,6$ & $55,3 \pm 2,4$ \\
Peso (kg) & $69,4 \pm 12,6$ & $64,7 \pm 6,4$ \\
Estatura (cm) & $158,0 \pm 4,5$ & $157,1 \pm 5,8$ \\
\hline
\end{tabular}

O programa de treinamento foi realizado durante quatro semanas, em duas sessões semanais, com duração de 50 minutos e intervalo de pelo menos 48 horas entre as sessões. As aulas foram ministradas em piscina aquecida com temperatura de $29^{\circ} \mathrm{C}$, com $1,40 \mathrm{~m}$ de profundidade, $20 \mathrm{~m}$ de comprimento e $12,5 \mathrm{~m}$ de largura.

Ambos os grupos (GTE e GTC) realizaram o mesmo programa de hidroginástica. Cada sessão era composta por quatro fases: fase 1) aquecimento - exercícios de alongamento (duração 10 minutos); fase 2) parte força - treinamento de força muscular (duração 20 minutos); fase 3) parte aeróbia - deslocamentos e exercícios de recreação (duração 10 minutos); fase 4) volta à calma - exercícios de alongamento e relaxamento (duração 10 minutos).

A fase 2 era composta por exercícios adaptados de protocolos de treinamento de força de resistência fixa: extensão e flexão de joelho, crucifixo em pé, remada, flexão e extensão de cotovelo, abdução de ombros e flexão anterior de ombros. Os exercícios foram realizados em três séries com 15-20 repetições.

Para o GTE, a fase 2 era realizada utilizando para aumento sobrecarga uma banda elástica de látex com 1,90m de comprimento e 3,5cm de diâmetro. O GTC realizava a fase 2 sem o incremento de sobrecarga adicional pela banda elástica, ou seja, os exercícios eram realizados somente com a resistência da água.

A intensidade do treinamento foi monitorada pela escala de percepção subjetiva de esforço proposta por Borg ${ }^{(12)}$. Durante a parte principal da aula (fase 2), os avaliados deveriam realizar as atividades sem ultrapassar e percepção máxima para o esforço "cansativo", indicada pelo no 15. Caso algum avaliado indicasse esforço superior a 15, este deveria diminuir a amplitude dos movimentos.

\section{Protocolos e medidas de aptidão física}

Antes e após o término do programa de treinamento, os indivíduos foram submetidos a uma bateria de testes motores composta por três exercícios, realizados na seguinte ordem: a) flexão de cotovelo: o sujeito sentado em uma cadeira com um halter de dois quilos na mão dominante deveria realizar o maior número de flexões e extensões em 30 segundos ${ }^{(13)}$; b) teste de impulsão vertical sem auxílio dos braços: o sujeito com os pés paralelos, corpo lateralmente à parede e os mem- 
bros superiores elevados verticalmente deveria saltar a maior altura possível(14); c) teste de levantar da cadeira em 30 segundos: o sujeito sentado em uma cadeira sem braços, com $43 \mathrm{~cm}$ de altura e braços cruzados na frente do tórax deveria ficar em pé e retornar para a posição inicial sentada o maior número de vezes em 30 segundos ${ }^{(13)}$. Enquanto o teste de impulsão vertical foi realizado três vezes (com intervalo de três minutos entre cada tentativa), o teste de flexão de cotovelo e o teste de levantar e sentar da cadeira foram realizados apenas uma vez, com intervalo de cinco minutos entre cada um.

\section{Tratamento estatístico}

Para a análise dos resultados foi utilizada estatística descritiva com média e desvio-padrão. Valores de normalidade dos dados foram determinados pelo teste de Shapiro-Wilks, optando-se pela estatística paramétrica. Para verificação do efeito do treinamento e da utilização da banda elástica foi utilizada análise de variância (ANOVA) por dois fatores (grupo e tempo). Quando verificado efeito significante em algum dos fatores, foi realizado o teste post hoc de Tukey para localização das diferenças. O nível de significância adotado foi de $P<0,05$. Todos os dados foram tratados no programa Statistica 7.0.

\section{RESULTADOS}

Quatro sujeitos do GTC não completaram o protocolo de treinamento no GTC, três por motivo de viagem e um por faltar mais de $25 \%$ das aulas. Na tabela 2 são apresentados os valores médios e desviopadrão do desempenho nos testes de medida da aptidão física pré e pós-treinamento para os grupos GTE e GTC. Os resultados da ANOVA indicaram que houve efeito significante do tempo de treinamento nos testes de flexão e extensão de cotovelo, impulsão vertical e teste motor de levantar e sentar na cadeira no GTE. Adicionalmente, nos testes de flexão de cotovelo e levantar e sentar da cadeira foi verificado efeito positivo da interação grupo $x$ treino $(P>0,01)$.

Tabela 2. Valores médios e desvio-padrão dos escores nos testes de flexão de cotovelo, impulsão vertical e levantar e sentar na cadeira, pré e pós-treinamento no GTE e GTC.

\begin{tabular}{|c|c|c|c|c|c|}
\hline Testes & $\begin{array}{c}\text { GTE } \\
(n=15)\end{array}$ & $\begin{array}{c}\text { GTC } \\
(n=11)\end{array}$ & Efeitos & $F$ & $P$ \\
\hline $\begin{array}{l}\text { Flexão de cotovelo } \\
\text { (repetições) }\end{array}$ & & & ANOVA & & \\
\hline Pré-treinamento & $21,8 \pm 3,5$ & $20,8 \pm 1,9$ & Grupo & 3,9 & 0,06 \\
\hline Pós-treinamento & $31,8 \pm 5,6 *$ & $24,8 \pm 3,9$ & Tempo & 47,6 & $<0,01 \dagger$ \\
\hline$\Delta \%$ & $+45,9$ & $+19,2$ & Grupo x tempo & 8,7 & $<0,01 \dagger$ \\
\hline $\begin{array}{l}\text { Impulsão vertical } \\
(\mathrm{cm})\end{array}$ & & & ANOVA & & \\
\hline Pré-treinamento & $14,5 \pm 4,7$ & $13,7 \pm 5,7$ & Grupo & 1,0 & 0,33 \\
\hline Pós-treinamento & $19,1 \pm 3,5$ * & $15,8 \pm 5,7^{*}$ & Tempo & 21,7 & $<0,01 \dagger$ \\
\hline$\Delta \%$ & $+31,7$ & $+15,3$ & Grupo x tempo & 2,9 & 0,10 \\
\hline $\begin{array}{l}\text { Levantar e sentar na } \\
\text { cadeira (repetições) }\end{array}$ & & & ANOVA & & \\
\hline Pré-treinamento & $17,3 \pm 2,5$ & $17,7 \pm 2,1$ & Grupo & 1,1 & 0,30 \\
\hline Pós-treinamento & $22,9 \pm 4,8 *$ & $19,3 \pm 3,1$ & Tempo & 30,1 & $<0,01 \dagger$ \\
\hline$\Delta \%$ & $+32,4$ & $+9,0$ & Grupo x tempo & 9,5 & $<0,01 \dagger$ \\
\hline
\end{tabular}

* Diferença significante em comparação com o pré-treinamento

† Efeito estatisticamente significante

\section{DISCUSSÃO}

A prática da hidroginástica é extremamente popular em adultos e idosos para a melhoria da aptidão física relacionada à saúde. De fato, estudos têm demonstrado que essa modalidade promove melhoria do $\mathrm{VO}_{2 \text { pico }}{ }^{(9)}$, flexibilidade ${ }^{(10)}$ e força muscular ${ }^{(9,10)}$.

Takeshima et al..$^{(9)}$ verificaram aumentos significantes no desempenho de salto horizontal e força de extensão e flexão de joelhos após um programa de hidroginástica de 12 semanas com três sessões semanais. Alves et al. ${ }^{(10)}$ observaram aumentos significantes no número de repetições nos testes de levantar e sentar da cadeira e flexão de cotovelo em idosos após 12 semanas com três sessões semanais.

No presente estudo não foi observado aumento da força após quatro semanas de treinamento de hidroginástica sem a utilização de elásticos (GTC). Resultados semelhantes aos observados por Taunton et al. ${ }^{(15)}$ que não verificaram aumentos significantes na força e resistência muscular após 12 semanas de treinamento de hidroginástica, o que pode ser explicado pela intensidade do treinamento utilizada no estudo não tendo sido suficiente para promover mudanças na força muscular.

Por outro lado, a intensidade utilizada no presente estudo foi relativamente elevada em função do critério estabelecido de 15 pontos da escala de percepção de esforço de Borg, que corresponde à classificação vigorosa ${ }^{(16)}$. Assim, possivelmente outros fatores, como o bom nível de treinamento dos sujeitos no início do programa de treinamento, juntamente com o curto período de intervenção, possam ter influenciado, pelo menos em parte, o comportamento da força no GTC.

Os resultados encontrados no GTE indicaram que a utilização de bandas elásticas durante as aulas de hidroginástica promoveu aumento da força muscular de membros superiores e inferiores em mulheres de meia-idade após quatro semanas de treinamento. Esses achados corroboram os resultados observados na literatura que verificaram aumentos significantes da força após o treinamento com bandas elásticas ${ }^{(5-8,17)}$.

A utilização de bandas elásticas consiste num dos tipos de resistência variável usada para o treinamento de força e apresenta como principal característica o aumento da resistência proporcional ao aumento da amplitude do movimento ${ }^{(18)}$. Alguns autores sugerem que a utilização da resistência elástica traria melhores resultados comparados com a resistência fixa ${ }^{(19)}$.

O treinamento com elástico apresenta vantagens como a versatilidade, uma vez que pode ser utilizado em inúmeros movimentos, e pela viabilidade econômica ${ }^{(4)}$. Além disso, a realização dos movimentos com bandas elásticas possibilita maior intensidade no componente excêntrico do movimento, conseqüentemente, otimizando os ganhos de força e massa muscular com o treinamento(5).

Os resultados do presente estudo indicaram efeito da interação grupo x tempo em favor do GTE nos dois testes que envolviam resistência muscular localizada (flexão de cotovelos e levantar e sentar na cadeira), ao passo que no exercício que envolvia potência, o efeito da interação grupo x treino não foi significante. Esses resultados sugerem que as adaptações promovidas pelo programa de treinamento foram específicas ao tipo realizado; o programa de treinamento de força (fase 2) era composto por exercícios de resistência muscular localizada (15-20 repetições).

Embora seja conhecida a importância da sobrecarga para aumento da força muscular, poucos estudos são encontrados na literatura em relação ao beneficio de implementos em exercícios na água. Considerando que a escassez de dados limite uma discussão mais profunda dos resultados do presente estudo, pudemos verifi- 
car benefícios neuromusculares em indivíduos adultos submetidos a programa de exercício na água com uso complementar de bandas elásticas, ressaltando aumento da força e resistência muscular de membros inferiores ao redor de 33\% para o grupo GTE e 14,5\% para o GTC, sugerindo ser uma estratégia possível e eficiente para complementar as adaptações neuromotoras promovidas em programa de exercícios na água.

No entanto, é importante ressaltar algumas limitações do presente estudo. Neste foi utilizada amostra com número relativamente reduzido. Entretanto, a realização de estudos que envolvam intervenção está atrelada a uma série de dificuldades, como espaço e horários disponíveis para o programa de treinamento, desistências, faltas de sujeitos, dentre outros. Por isso, muitas vezes a utilização de muitos sujeitos é inviável. Outra limitação do estudo refere-se à adaptação de protocolos de treinamento desenvolvidos para situações de resistência fixa ${ }^{(20)}$ utilizando bandas elásticas, procedimento que ainda não apresenta fundamentação teórica.

\section{CONCLUSÃO}

Os resultados encontrados no presente estudo sugerem que a associação do treinamento de força realizado por meio de bandas elásticas e a hidroginástica promoveram aumentos significantes na força muscular de membros inferiores e superiores dos sujeitos. Dessa forma, por se tratar de um método versátil e viável economicamente, sugerimos incorporar os exercícios com bandas elásticas durante as sessões de hidroginástica para o aumento da força muscular.

Todos os autores declararam não haver qualquer potencial conflito de interesses referente a este artigo.

\section{REFERÊNCIAS BIBLIOGRÁFICAS}

1. Matsudo SMM, Matsudo VKR, Araújo TL. Perfil do nível de atividade física e capacidade funcional de mulheres maiores de 50 anos de idade de acordo com a idade cronológica. Revista Brasileira de Atividade Física e Saúde 2001;6:12-24.

2. Frontera WR, Hughes VA, Fielding RA, Fiatarone MA, Evans WJ, Roubenoff R. Aging of skeletal muscle: a 12-yr longitudinal study. J Appl Physiol 2000;88(4):1321-6.

3. American College of Sports Medicine Position Stand. Exercise and physical activity for older adults. Med Sci Sports Exerc 1998;30(6):992-1008

4. Azevedo FM, Negrão Filho RF, Carvalho ACA. Atividade elétrica gerada pelo músculo bíceps braquial em diferentes ângulos e resistências. Revista de Fisioterapia da Universidade de São Paulo 1999:6(suppl):39-39

5. Treiber FA, Lott J, Duncan J, Slavens G, Davis H. Effects of Theraband and lightweight dumbbell training on shoulder rotation torque and serve performance in college tennis players. Am J Sports Med 1998;26(4):510-5

6. Mikesky AE, Topp R, Wigglesworth JK, Harsha DM, Edwards JE. Efficacy of a home-based training program for older adults using elastic tubing. Eur J Appl Physiol Occup Physiol 1994;69(4):316-20.

7. Capodaglio P, Ferri A, Scaglloni G. Effects of a partially supervised training program in subjects over 75 years of age. Aging Clin Exp Res 2005;17(3):174-80.

8. Vanbiervliet W, Pelissier J, Ledermann B, Kotzki N, Benaim C, Herisson C. Strength training with elastic bands: measure of its effects in cardiac rehabilitation after coronary diseases. Ann Readapt Med Phys 2003;46(8):545-52.

9. Takeshima N, Rogers ME, Watanabe E, Brechue WF, Okada A, Yamada T, et al. Water-based exercise improves health-related aspects of fitness in older women. Med Sci Sports Exerc 2002;34(3):544-51.

10. Alves RV, Mota J, Costa MC, Alves JGB. Aptidão física relacionada à saúde de idosos: influência da hidroginástica. Revista Brasileira de Medicina do Esporte 2004;10(1):31-7.
11. Kihlstrand M, Stenman B, Nilsson S, Axelsson O. Water-gymnastics reduced the intensity of back/low back pain in pregnant women. Acta Obstet Gynecol Scand 1999;78(3):180-5.

12. Borg GA. Psychophysical bases of perceived exertion. Med Sci Sports Exerc 1982;14(5):377-81.

13. Rikli R, Jones J. Development and validation of a functional fitness test for community-residing older adults. J Aging Phys Act 1999;7:129-81.

14. Soares J, Sessa M. Medidas de força muscular. In: Matsudo V, editor; Testes em ciências do esporte. São Caetano do Sul: CELAFISCS; 1995. p.52-72.

15. Taunton JE, Rhodes EC, Wolski LA, Donelly M, Warren J, Elliot J, et al. Effect of land-based and waterbased fitness programs on the cardiovascular fitness, strength and flexibility of women aged 65-75 years. Gerontology 1996;42(4):204-10.

16. Warburton DE, Nicol CW, Bredin SS. Prescribing exercise as preventive therapy. CMAJ 2006;174(7):961-74

17. Wallace BJ, Winchester JB, McGuigan MR. Effects of elastic bands on force and power characteristics during the back squat exercise. J Strength Cond Res 2006;20(2):268-72.

18. Benatti LN. Estudo da força gerada pelo grupo muscular ísquio-tibial em exercícios resistidos [Dissertação de mestrado]. Escola de Engenharia em São Carlos, Faculdade de Medicina em Ribeirão Preto, Instituto de Química de São Carlos: Universidade de São Paulo; 2005.

19. Hintermeister RA, Lange GW, Schultheis JM, Bey MJ, Hawkins RJ. Electromyographic activity and applied load during shoulder rehabilitation exercises using elastic resistance Am J Sports Med 1998;26(2):210-20.

20. Azevedo FM. Estudo da força e da atividade elétrica gerada pelo músculo quadríceps femoral submetido à exercícios com resistÊncia elástica. [Dissertação de mestrado]. Escola de Engenharia em São Carlos, Faculdade de Medicina em Ribeirão Preto, Instituto de Química de São Carlos: Universidade de São Paulo; 2003. 